\title{
Magnetic Properties and Relaxation of Vanadium Monolayer on Pd(001) Surface
}

\author{
Kalpana K. Landge, Beata Białek, and Jae II Lee* \\ Department of Physics, Inha University, Incheon 402-751, Korea
}

(Received 26 March 2010, Received in final form 26 May 2010, Accepted 27 May 2010)

\begin{abstract}
We investigated the magnetism of vanadium monolayers on a Pd(001) surface. The electronic structure and the magnetic properties of the $\mathrm{V} / \mathrm{Pd}(001)$ system were determined with the use of the full-potential linearized augmented plane-wave method within the general gradient approximation. Three magnetic configurations were studied: non-, ferro-, and antiferromagnetic. From the total energy calculations, we found that the V/Pd(001) system is the most stable in the antiferromagnetic configuration. The importance of relaxation on the magnetic properties of the systems was also studied. It was found that the $\operatorname{Pd}(001)$ surface covered with a $V$ monolayer undergoes considerable relaxation in which the spacing between Pd layers increases in all three magnetic configurations. Contrary to the Pd interlayer spacing, the distance between the $\mathrm{V}$ overlayer and the topmost Pd layer is reduced. The interlayer spacing between the $\mathrm{V}$ overlayer and the Pd surface layer is the largest for the antiferromagnetic configuration. In the relaxed antiferromagnetic structure, the magnitude of the calculated magnetic moments on the $V$ atoms was $1.31 \mu_{\mathrm{B}}$. The presence of the vanadium monolayer does not affect the paramagnetic properties of the $\operatorname{Pd}(001)$ surface.
\end{abstract}

Keywords : magnetism, multilayer relaxation, vanadium, palladium

\section{Introduction}

Magnetic properties of 3d transition metal (TM) adatoms and monolayers (MLs) have been subjects of interest for many years [1-5]. The main reason for this interest is that the $3 \mathrm{~d}$ elements in the form of lowdimensional structures exhibit different magnetic properties than their bulk structures. With the possibility of producing low-dimensional structures with desired geometries on select substrates, the magnetic properties of materials may be tailored on demand.

One of the $3 d$ elements of interest is vanadium. Its impurities, adatoms, small clusters, and monolayers deposited on surfaces of different nonmagnetic materials have been studied widely [1-3,5-7]. Vanadium on Pd surfaces is particularly interesting because of rather small misfits between their lattice parameters, which allows $\mathrm{V}$ to be epitaxially grown on a $\mathrm{Pd}$ substrate. The $\mathrm{V} / \mathrm{Pd}$ system is also of interest because the $\mathrm{Pd}$ is a paramagnetic element on the verge of being magnetic, $[8,9]$ and its presence at the interface with $\mathrm{V}$ may be sufficient to induce a sizeable magnetic moment. A systematic study

*Corresponding author: Tel: +82-32-860-7654

Fax: +82-32-872-7562, e-mail: jilee@inha.ac.kr based on the full potential linearized augmented plane wave method (FLAPW) was carried out for $3 \mathrm{~d}, 4 \mathrm{~d}$ and $5 \mathrm{~d}$ MLs on noble metals; it was predicted that the antiferromagnetic (AF) configuration for $\mathrm{V} M L$ on the paramagnetic noble metal substrates is preferable [2, 3]. It was discovered that even though noble metal substrates do not generally affect the magnetic properties of most $3 \mathrm{~d}$ metals, a significant effect is observed in the case of $\mathrm{V}$ monolayers. Palladium is strongly exchange-enhanced and, therefore, a magnetic overlayer, such as V, should induce considerable spin polarization in the underlying Pd layers. The $\mathrm{V}$ atoms in an overlayer atop a $\operatorname{Pd}(001)$ surface were calculated to have a $1.89 \mu_{\mathrm{B}}$ magnetic moment [2]. A few years later, Stepanyuk et al. reported systematic $a b$ initio calculations for various $3 \mathrm{~d}$ TM MLs on $\mathrm{Pd}(001)$ surfaces [5]. According to their results, $\mathrm{V}$ atoms in a monolayer have a very small $\left(-0.01 \mu_{\mathrm{B}}\right)$ magnetic moment. Discrepancies between the reported results may come from various factors: the method of calculations; the chosen lattice constant; and/or the geometry of the V monolayer on the $\operatorname{Pd}(001)$ substrate. Also, a very important factor may be the atomic relaxation of the system. This possibility was very shortly qualitatively discussed in Ref. [5]. Until now, however, no report on the magnetic properties of a $\mathrm{V} / \mathrm{Pd}(001)$ system in which the relaxation problem has 
been considered explicitly has been presented.

In this paper, we describe the results of an $a b$ initio study of the magnetic properties of $\mathrm{V}$ monolayers on a $\operatorname{Pd}(001)$ surface with a strong focus on the relaxation phenomena in the system.

\section{Calculational Method}

The investigated $\mathrm{V} / \mathrm{Pd}(001)$ system was modeled by a nine-layer slab, as shown in Fig. 1. The slab consisted of seven layers of Pd parallel to the (001) surface of the Pd fcc structure and a monolayer of $\mathrm{V}$ attached to the both sides of the Pd slab. The lattice constant of the Pd fcc bulk was 7.35 a.u., and therefore the initial interlayer spacing in the slab was assumed to equal 3.675 a.u. Since at a low temperature vanadium crystallizes in a bcc structure with the lattice constant of 5.81 a.u., we arbitrarily chose the interlayer spacing between the $\mathrm{V}$ monolayer and the topmost Pd layer to be 3.658 a.u.

Three magnetic configurations of the system were considered: the nonmagnetic (NM), ferromagnetic (FM), and $c(2 \times 2)$ antiferromagnetic (AFM) structures. Spin ordering in the AFM superstructure is also shown in Fig. 1.

The Kohn-Sham equation was solved self-consistently with the use of the all-electron FLAPW method [10,11] based on the generalized gradient approximation (GGA) [12] for the exchange-correlation potential. Lattice harmonics with $l \leq 8$ were employed to expand the charge density, potential, and wave functions within the muffintin (MT) radii of 2.20 and 2.30 a.u. for the $\mathrm{V}$ and $\mathrm{Pd}$ atoms, respectively. An energy cutoff of $16 \mathrm{Ry}$ was em-

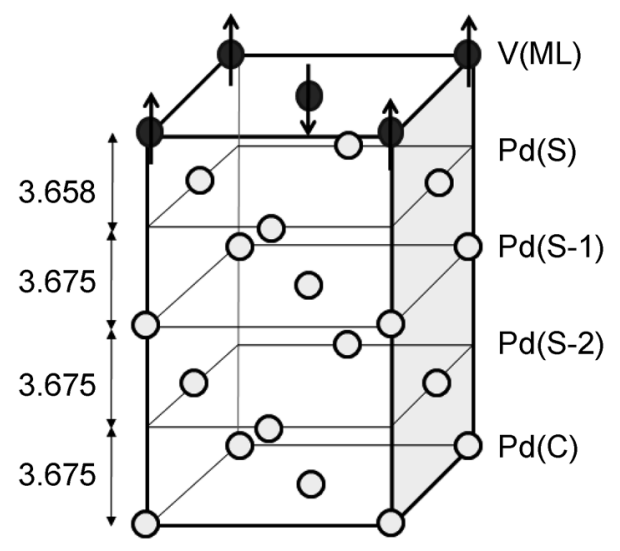

Fig. 1. A schematic view of half of a 9-layer $\mathrm{V} / \mathrm{Pd}(001)$ slab with the initial interlayer spacing indicated (the values are given in a.u.). Pd atoms are shown as opened circles, while $\mathrm{V}$ atoms are represented by filled dark circles. The arrows indicate magnetic ordering in the top $\mathrm{V}$ monolayer, as was assumed for the AFM structure. ployed for the basis set. A 100 Ry star function cutoff was used for depicting the charge density and potential in the interstitial region. Integrations inside the two dimensional (2D) Brillouin zone (BZ) were replaced by summation over $24 k$-points inside the irreducible wedge of the 2D BZ. All core electrons were treated fully relativistically, while valence states were treated scalar-relativistically, i.e. without spin-orbit coupling. Self-consistency was assumed when the difference between the input and output charge (spin) densities was less than $1.0 \times 10^{-4}$ electrons/a.u. ${ }^{3}$.

The $V / P d(001)$ system was allowed to relax in the vertical direction so the optimized values of the interlayer spacing could be obtained and the structural changes in the 7-layer Pd(001) slab due to the presence of a V ML could be studied. Multilayer relaxation was performed through the total energy and atomic force calculations. The equilibrium configuration was assumed when the atomic force on each atom was smaller than $1.5 \mathrm{mRy} / \mathrm{a}$.u. For comparison, the calculations were also performed for a 7-layer $\operatorname{Pd}(001)$ slab not covered with the V monolayer.

\section{Result and Discussion}

Before presenting the results concerning the electronic structures of the $\mathrm{V} / \mathrm{Pd}(001)$ system, we shall discuss the relaxation effect on the 7-layer NM $\operatorname{Pd}(001)$ slab. The calculated changes in the interlayer spacing in the $\operatorname{Pd}(001)$ slab with respect to the unrelaxed structure did not exceed 1 percent. All the layers relaxed in the outward direction, thus expanding the total thickness of the slab by 0.25 a.u. The change in the interlayer spacing was not very large, however, which is in agreement with both the other calculations [13] and another experiment [14]. The exact values concerning the changes are given in Table 1. Even though the relaxation effect was small, it had a certain influence on the charge distribution between the atoms in the slab, especially in the topmost layer. The calculated number of the $l$-decomposed electrons inside the Pd MT spheres indicates that the number of $d$ electrons is slightly larger in the relaxed structure (7.89 vs. 7.88 in the unrelaxed structure). At the same time the number of $p$ electrons within the Pd MT decreased from 0.14 in the unrelaxed $\operatorname{Pd}(001)$ to 0.13 in the relaxed structure. The difference in the distribution of electrons in the relaxed slab may be important for the electronelectron interactions in the $\mathrm{V} / \mathrm{Pd}(001)$ system.

From the total energy calculations done for both unrelaxed and relaxed $\mathrm{V} / \mathrm{Pd}(001)$ systems in the three magnetic configurations, we found that the most stable configuration is the AFM one. For the relaxed system, its total energy in the AFM configuration was $159 \mathrm{meV}$ 
Table 1. The interlayer spacing $\left(\mathrm{d}_{\mathrm{rlx}}\right)$ in the relaxed $\operatorname{Pd}(001)$ clean slab and the slab covered with the V ML, along with their changes relative to the unrelaxed $\mathrm{Pd}(001)$ surface.

\begin{tabular}{lcccccccc}
\hline \hline & \multicolumn{2}{c}{$\mathrm{Pd}(001)$} & \multicolumn{2}{c}{ V/Pd NM } & \multicolumn{2}{c}{ V/Pd FM } & \multicolumn{2}{c}{ V/Pd AFM } \\
\cline { 2 - 9 } & $\mathrm{d}_{\mathrm{rlx}}$ & $\%$ & $\mathrm{~d}_{\mathrm{rlx}}$ & $\%$ & $\mathrm{~d}_{\mathrm{rlx}}$ & $\%$ & $\mathrm{~d}_{\mathrm{rlx}}$ & $\%$ \\
\hline V(ML)-Pd(S) & & & 3.439 & -5.99 & 3.439 & -5.99 & 3.470 & -5.14 \\
Pd(S)-Pd(S-1) & 3.677 & 0.05 & 3.905 & 6.26 & 3.909 & 6.12 & 3.948 & 7.43 \\
Pd(S-1)-Pd(S-2) & 3.676 & 0.03 & 3.787 & 3.05 & 3.798 & 3.35 & 3.843 & 4.57 \\
Pd(S-2)-Pd(C) & 3.675 & 0 & 3.748 & 1.99 & 3.772 & 2.64 & 3.806 & 3.56 \\
\hline
\end{tabular}

lower than that of the FM state and $171 \mathrm{meV}$ lower than that of the system in NM configuration. The differences between the total energy of the systems in the NM and FM state was as small as $13 \mathrm{meV}$, which may explain why in some of the previous studies of the $\mathrm{V} / \mathrm{Pd}(001)$ surfaces there was disagreement about the preferable magnetic configuration.

As can be seen in Fig. 2 and through the data collected in Table 1 , covering the $\operatorname{Pd}(001)$ slab with the $\mathrm{V}$ ML has a large effect on the Pd slab structure. In any magnetic configuration, the interlayer spacing in the $\operatorname{Pd}(001)$ slab

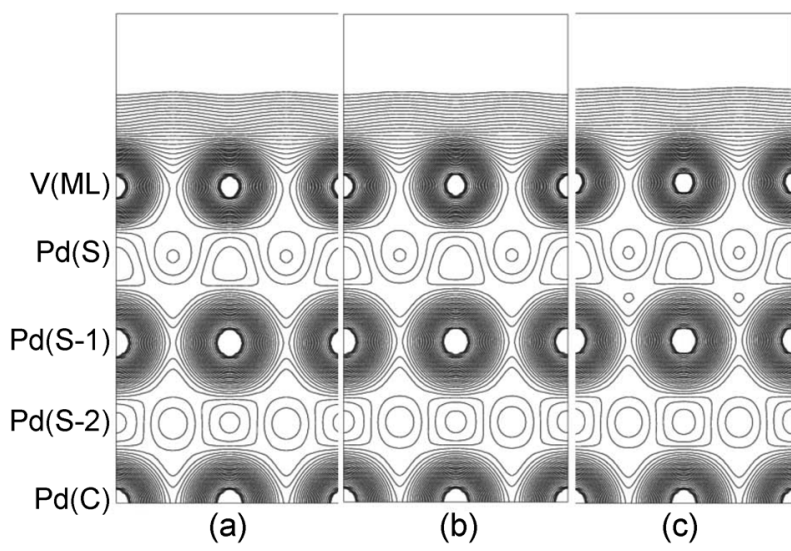

Fig. 2. Contours of charge density in a (110) plane of V/ Pd(001) for (a) NM, (b) FM, and (c) AFM configuration. The lowest contour starts from $1 \times 10^{-4}$ electrons/ a.u. ${ }^{3}$ and subsequent lines successively increase by a factor of $\sqrt{2}$. changes considerably. Due to the relaxation caused by the presence of the V ML, the distance between the $\operatorname{Pd}(\mathrm{S})$ and $\mathrm{Pd}(\mathrm{S}-1)$ layers increases by $6.26,6.12$, and $7.43 \%$ in the NM, FM, and AFM configurations, respectively. Also the spacing between the $\mathrm{Pd}(\mathrm{S}-1)$ and $\mathrm{Pd}(\mathrm{S}-2)$ layers changes greatly. The presence of the V ML affects even the deeper $\mathrm{Pd}(\mathrm{S}-2)$ layer, which also moves upward. In the AFM configuration, the distance between the center and S-2 layers changes by more than $4 \%$. This may suggest that the slab chosen for the study should be thicker. The optimized distances between the V ML and the $\operatorname{Pd}(\mathrm{S})$ layer are much smaller than the average of the unrelaxed values of the bcc $\mathrm{V}$ and fcc Pd lattice constants. In both the $\mathrm{NM}$ and FM V/Pd(001) systems, the V ML is only 3.439 a.u. above the $\mathrm{Pd}$ surface layer, while in the AFM configuration the $\mathrm{V}-\mathrm{Pd}(\mathrm{S})$ distance is slightly larger than that in the NM and FM configurations. This relative expansion of the distance between the $\mathrm{V}$ and the $\operatorname{Pd}(\mathrm{S})$ layers in the AFM configuration may be explained through the analysis of the numbers of $l$-decomposed electrons within each MT sphere.

Interestingly, even though relaxation was noticeable in the PM configuration, the number of $l$-decomposed electrons within each MT sphere of the two systems, unrelaxed NM and relaxed NM systems, did not change much. Since the NM configuration appeared to be much less stable than the other two, i.e. the FM and AFM ones, we will not present the detailed result for the NM case.

Table 2 and Table 3 contain the numbers of $l$-decomposed

Table 2. Number of $l$-decomposed electrons within each MT sphere of the V ML and $\mathrm{Pd}(\mathrm{S})$ layer of the unrelaxed and relaxed V/ $\mathrm{Pd}(001)$ systems in the FM configuration, along with the local magnetic moment on each atom.

\begin{tabular}{cccccr}
\hline \hline & \multicolumn{5}{c}{ Unrelaxed } \\
\hline Atom (layer) & $\mathrm{s}(\uparrow / \downarrow)$ & $\mathrm{p}(\uparrow / \downarrow)$ & $\mathrm{d}(\uparrow / \downarrow)$ & total $(\uparrow / \downarrow)$ & magnetic moment $\left[\mu_{\mathrm{B}}\right]$ \\
\hline $\mathrm{V}(\mathrm{ML})$ & $0.11 / 0.10$ & $0.08 / 0.06$ & $2.00 / 0.77$ & $2.19 / 0.93$ & 1.26 \\
$\mathrm{Pd}(\mathrm{S})$ & $0.15 / 0.16$ & $0.12 / 0.12$ & $3.91 / 3.88$ & $4.20 / 4.16$ & 0.04 \\
\hline \multicolumn{7}{c}{ Relaxed } & & \\
\hline Atom (layer) & $\mathrm{s}(\uparrow / \downarrow)$ & $\mathrm{p}(\uparrow / \downarrow)$ & $\mathrm{d}(\uparrow / \downarrow)$ & total $(\uparrow / \downarrow)$ & magnetic moment $\left[\mu_{\mathrm{B}}\right]$ \\
\hline $\mathrm{V}(\mathrm{ML})$ & $0.11 / 0.10$ & $0.08 / 0.07$ & $1.66 / 1.10$ & $1.86 / 1.27$ & 0.59 \\
$\mathrm{Pd}(\mathrm{S})$ & $0.16 / 0.16$ & $0.12 / 0.12$ & $3.89 / 3.89$ & $4.18 / 4.18$ & 0.00 \\
\hline
\end{tabular}


Table 3. Number of $l$-decomposed electrons within each MT sphere of the V ML and $\mathrm{Pd}(\mathrm{S})$ layer for the unrelaxed and relaxed $\mathrm{V} /$ $\operatorname{Pd}(001)$ systems in the AFM configuration, along with the local magnetic moment of each atom.

\begin{tabular}{|c|c|c|c|c|c|}
\hline \multicolumn{6}{|c|}{ Unrelaxed } \\
\hline Atom (layer) & $\mathrm{s}(\uparrow / \downarrow)$ & $\mathrm{p}(\uparrow / \downarrow)$ & $\mathrm{d}(\uparrow / \downarrow)$ & $\operatorname{total}(\uparrow / \downarrow)$ & magnetic moment $\left[\mu_{\mathrm{B}}\right]$ \\
\hline \multirow[t]{2}{*}{$\mathrm{V}(\mathrm{ML})$} & $0.11 / 0.10$ & $0.07 / 0.07$ & $2.12 / 0.65$ & $2.29 / 0.82$ & 1.48 \\
\hline & $0.09 / 0.10$ & $0.07 / 0.07$ & $0.65 / 2.12$ & $0.82 / 2.30$ & -1.48 \\
\hline \multirow[t]{2}{*}{$\operatorname{Pd}(\mathrm{S})$} & $0.15 / 0.15$ & $0.12 / 0.12$ & $3.90 / 3.89$ & $4.18 / 4.18$ & 0.00 \\
\hline & $0.15 / 0.15$ & $0.12 / 0.12$ & $3.90 / 3.90$ & $4.18 / 4.18$ & 0.00 \\
\hline \multicolumn{6}{|c|}{ Relaxed } \\
\hline Atom (layer) & $\mathrm{s}(\uparrow / \downarrow)$ & $\mathrm{p}(\uparrow / \downarrow)$ & $\mathrm{d}(\uparrow / \downarrow)$ & $\operatorname{total}(\uparrow / \downarrow)$ & magnetic moment $\left[\mu_{\mathrm{B}}\right]$ \\
\hline \multirow[t]{2}{*}{$\mathrm{V}(\mathrm{ML})$} & $0.11 / 0.10$ & $0.07 / 0.08$ & $2.04 / 0.74$ & $2.23 / 0.92$ & 1.31 \\
\hline & $0.10 / 0.11$ & $0.08 / 0.07$ & $0.74 / 2.04$ & $0.92 / 2.23$ & -1.31 \\
\hline \multirow[t]{2}{*}{$\operatorname{Pd}(\mathrm{S})$} & $0.16 / 0.16$ & $0.11 / 0.11$ & $3.90 / 3.89$ & $4.18 / 4.17$ & 0.01 \\
\hline & $0.16 / 0.16$ & $0.11 / 0.11$ & $3.90 / 3.89$ & $4.18 / 4.17$ & 0.01 \\
\hline
\end{tabular}

electrons within MT spheres of the FM and AFM V/ $\operatorname{Pd}(001)$ systems, respectively. Only the data concerning the $\mathrm{V}$ overlayer and $\mathrm{Pd}$ surface layer are shown, since the deeper layers of Pd did not exhibit magnetic properties according to our calculations. The values calculated for both the unrelaxed and relaxed slabs are included. The total number of electrons within the V MT sphere is 3.123 , and the number of electrons within the $\operatorname{Pd}(\mathrm{S}) \mathrm{MT}$ sphere is 8.360 in the relaxed FM system. The respective values for the relaxed AFM system are 3.141 and 8.356. It is $\operatorname{Pd}(\mathrm{S}) p$-electrons that go into the interstitial region in the AFM V/Pd(001) system. Due to this redistribution of electrons, the interlayer spacing increases. The electron delocalization is larger in the relaxed AFM V/Pd(001) system than in the NM and FM ones, as seen in Fig. 1, in the area between the $\mathrm{Pd}(\mathrm{S}-1)$ and $\mathrm{Pd}(\mathrm{S})$ layers.

As seen in Table 2, the relaxation of the system is of great importance for obtaining the most accurate values of the local MMs. This is especially clear in the case of the FM configuration: the calculated values of the magnetic moments on the $\mathrm{V}$ atom in the unrelaxed $\mathrm{V} / \mathrm{Pd}(001)$ is overestimated by $0.67 \mu_{\mathrm{B}}$, as compared to the value obtained for the relaxed system. The $\mathrm{Pd}$ slab in the relaxed $\mathrm{FM} \mathrm{V/Pd(001)} \mathrm{structure} \mathrm{remained} \mathrm{paramagnetic.}$

In the case of the AFM configuration of the $\mathrm{V} / \mathrm{Pd}(001)$ system, we also noticed the importance of the relaxation. The calculated magnitude of the local MM on the $\mathrm{V}$ atoms of the unrelaxed structure was $1.48 \mu_{\mathrm{B}}$, while it was $1.31 \mu_{\mathrm{B}}$ in the relaxed structure. Similarly, as in the FM configuration, the distribution of the $\mathrm{V}$ d-electrons changes slightly, which lowers the total energy of the system and stabilizes it energetically. As in all other cases, the $\operatorname{Pd}(001)$ slab remains nonmagnetic.

Blügel et al. noticed that the calculated local magnetic moments on $3 \mathrm{~d}$ metal atoms forming an overlayer on a
$\operatorname{Pd}(001)$ are almost the same for the FM and AFM configurations [2]. The only exception in the $3 \mathrm{~d}$ metal series was vanadium. The value of the magnetic moment of the AFM V on the $\operatorname{Pd}(001)$ plate calculated by Blügel et al. was only $0.51 \mu_{\mathrm{B}}$, which is much smaller than that obtained for the FM configuration $\left(1.39 \mu_{\mathrm{B}}\right)$ [2]. According to the results of our calculation, the magnetic moments on the $\mathrm{V}$ atoms in the unrelaxed FM and AFM configurations are also similar in magnitude: 1.26 and $1.48 \mu_{\mathrm{B}}$ for the FM and AFM configurations, respectively. Blügel et al. did not consider the relaxation. After the systems were allowed to relax, the value of the calculated magnetic

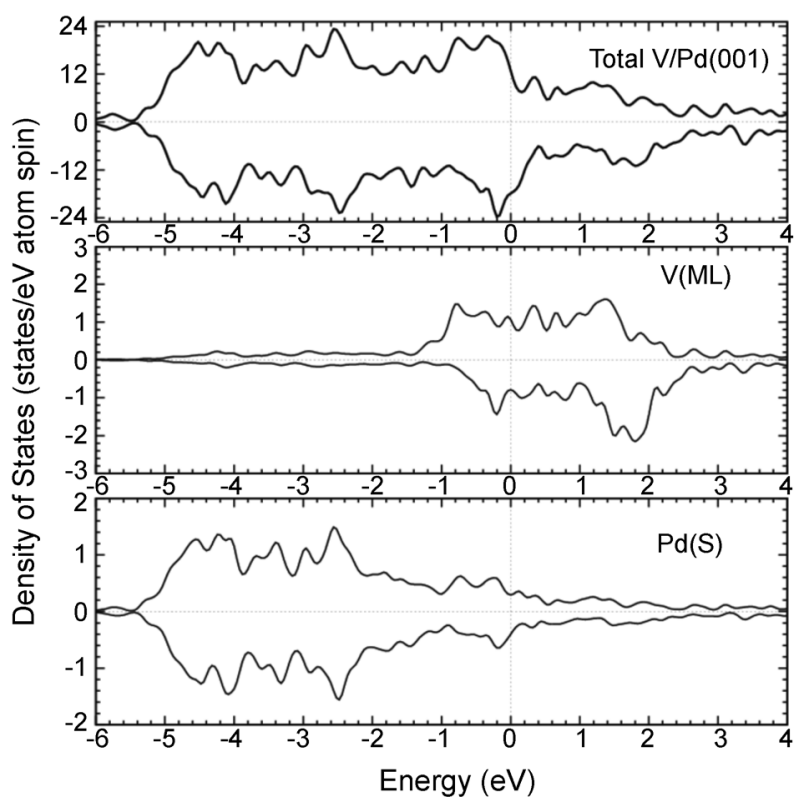

Fig. 3. The spin-polarized layer-projected density of states in the FM V/Pd(001) system. The negative values represent spindown electrons and the Fermi levels are set to zero. 


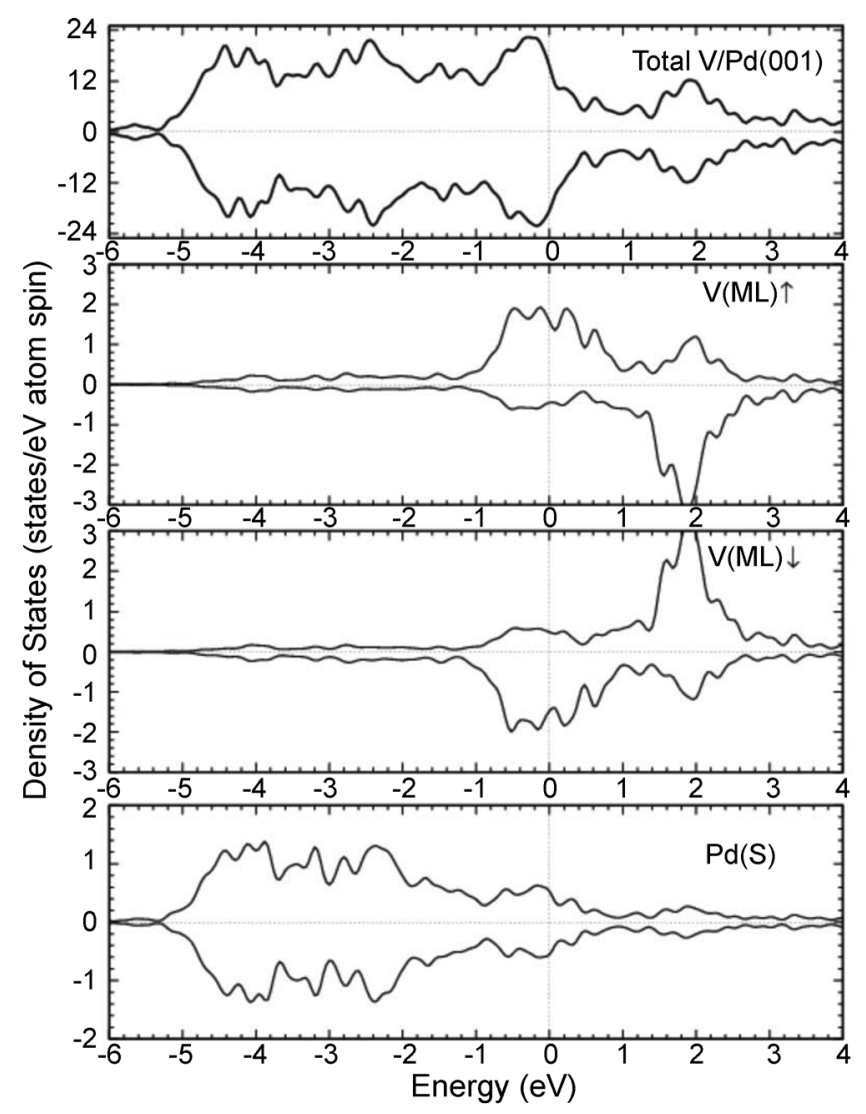

Fig. 4. The spin-polarized layer-projected density of states in the AFM V/Pd(001) system. The negative values represent spin-down electrons and the Fermi levels are set to zero.

moment in the $\mathrm{AFM} \mathrm{V/Pd(001)} \mathrm{configuration} \mathrm{became}$ $1.31 \mu_{\mathrm{B}}$, which is $0.72 \mu_{\mathrm{B}}$ larger than the relaxed FM V/ $\operatorname{Pd}(001)$ system. This again confirms that the relaxation is important for determining the electronic structures and magnetism of the V ML on the $\operatorname{Pd}(001)$ surface.

In Figs. 3 and 4, the spin-polarized layer-projected density of states (DOS) are given for the respective FM and $\mathrm{AFM} \mathrm{V/Pd(001)} \mathrm{systems.} \mathrm{The} \mathrm{negative} \mathrm{values} \mathrm{represent}$ minority electrons; the Fermi levels were set to zero.

The overall shapes of the total occupied DOS of the FM and AFM V/Pd(001) configurations are similar to each other, especially below $-1 \mathrm{eV}$. Slightly sharper peaks are seen in the FM total DOS picture at $-0.4 \mathrm{eV}$ for majority states and at $-0.2 \mathrm{eV}$ for minority states, which suggests that the occupied states near the Fermi level are more localized in the FM than in the AFM structure. There is also a peak of majority electron states present at $-0.8 \mathrm{eV}$ in the FM system and no similar feature is seen in the AFM system. From the layer-projected DOS drawn in Fig. 3, one can seen that the electrons confined in the $\mathrm{V}$ overlayer hybridized with those in the $\operatorname{Pd}(\mathrm{S})$ layer. The hybridization is not as strong within the AFM system, as seen in Fig. 4, because the $\mathrm{V}(\mathrm{ML})$ and $\mathrm{Pd}(\mathrm{S})$ layers are at a larger distance from each other than in the FM structure, and so the interactions between the $3 \mathrm{~d}$ electrons of $\mathrm{V}$ and the $4 \mathrm{~d}$ electrons of $\mathrm{Pd}$ are weaker. This is consistent with the analysis of the charge density distributions within the FM and AFM V/Pd(001) systems.

\section{Conclusions}

We investigated the magnetism of vanadium monolayers on a $\operatorname{Pd}(001)$ surface with special concern for the importance of structural relaxation of the investigated systems. Three magnetic configurations were considered, i.e. non-, ferro-, and antiferromagnetic. Due to the presence of the V ML on top of the $\operatorname{Pd}(001)$ slab, the slab undergoes considerable outward relaxation. In all three configurations, the interlayer distances between the Pd layers became larger as compared with that of the clean $\operatorname{Pd}(001)$ surface. The effect of the relaxation is the strongest in the most stable AFM configuration. Unlike the Pd layers, the top V ML relaxes inward in every magnetic configuration. The calculated values of the local magnetic moments of the $\mathrm{V}$ atoms are $0.59 \mu_{\mathrm{B}}$ and $1.31 \mu_{\mathrm{B}}$ for the FM and AFM configurations, respectively. The substrate $\operatorname{Pd}(001)$ slab remains nonmagnetic in the $\mathrm{V} / \mathrm{Pd}(001)$ system.

\section{Acknowledgement}

This work was supported by Inha University Research Funds.

\section{References}

[1] C. L. Fu, A. J. Freeman, and T. Oguchi, Phys. Rev. Lett. 54, 2700 (1985).

[2] S. Blügel, M. Weinert, and P. H. Dederichs, Phys. Rev. Lett. 60, 1077 (1988).

[3] S. Blügel, B. Drittler, R. Zeller, and P. H. Dederichs, Appl. Phys. A 49, 547 (1989).

[4] S. J. Youn and S. C. Hong, J. Magnetics 13, 140 (2008).

[5] V. S. Stepanyuk, W. Hergert, K. Wildberger, R. Zeller and P. H. Dederichs, Phys. Rev. B 53, 2121 (1996).

[6] T. Bryk, D. M. Bylander, L. Kleinman, Phy. Rev. B 61, R3780 (2000).

[7] A. A. Ramanathan, J. M. Khalifeh, B. A. Hamad, J. Magn. Magn. Mater. 321, 3804 (2009).

[8] S. C. Hong, J. I. Lee and R. Wu, Phy. Rev. B 75, 172402 (2007).

[9] S. C. Hong and J. I. Lee, J. Korean Phys. Soc. 52, 1099 (2008). 
[10] E. Wimmer, H. Krakauer, M. Weinert, and A. J. Freeman, Phys. Rev. B 24, 6864 (1981).

[11] M. Weinert, E. Wimmer, and A. J. Freeman, Phys. Rev. B 26, 4571 (1982).

[12] J. P. Perdew, K. Burke, and M. Ernzerhof, Phys. Rev.
Lett. 77, 3865 (1996).

[13] J. M. Zhang, Y. Shu and K. W. Xu, Solid State Commun. 137, 441 (2006).

[14] J. Quinn, Y. S. Li, D. Tian, H. Li, F. Jona, and P. M. Marcus, Phys. Rev. B 42, 11348 (1990). 